\title{
Rheumatology in the future
}

In the year 2001

Personal experience suggests a lugubrious rheumatological scenario 10 years hence. If the main difference between greybeards and neophytes is that, for the former, deja vu precedes the conference, why should anything have changed fundamentally in such a relatively short time span? Such a view would reflect a meanness of spirit, which must be instantly rejected.

There are two main dilemmas facing our specialty, one intellectual and the other practical. The intellectual problem is to discern why genetic predisposition to so many rheumatic diseases affects relatively few of those at risk. The second is to devise a strategy for turning scientific knowledge to therapeutic effect.

The key to understanding the scientific problem is to define what are now loosely described as environmental factors. In all probability, reactive arthritis will prove to be the prime 1991 example of what will become a general truth. Strategies for coping with common infectious agents have produced an evolutionary price in terms of immunopathological consequences. By 2001 we will know in precise detail why the inheritance of certain HLA antigens confers susceptibility to such infections and the same is likely to be true of 'autoimmune diseases' initiated by more protean infections. Nor will these principles be any less applicable to metabolic disorders, such as degenerative joint disease, which clearly reflect a similar interaction between phenotype and environmental, albeit non-infectious, stimuli.

For a variety of technical, ethical, and logistic reasons the therapeutic response will not entail 'genetic engineering'. It will require a full analysis of the infectious, dietary, mechanical, hormonal, and personal factors which precipitate rheumatic disorders. The relevant interviews will not be held in rheumatology clinics; they will be conducted in preventive health clinics. The main subject of contention will be the incentive those responsible for primary health care should receive for completing a $100 \%$ survey of their practice.

By 2001, rheumatologists will have proved equal to the splendid opportunity of solving this scientific and practical challenge, thereby turning a political shibboleth into a reality in their sphere of activity. Of course, all this has been said before: 'Fortunately, there is now a solution of the problem of providing for adaptability, and it goes some way towards making up for these inborn inequalities and imperfections of men ... the newer solution is to improve the environment ....,

A M DENMAN Clinical Research Centre Northwick Park Hospital Watford Road

Middlesex HAI 3 UI

1 Medawar P. In: Pyke D, ed. The threat and the glory. Oxford: Oxford University Press, 1990: 143.

\section{An epidemiological view}

LUMPING OR SPLITTING IN THE 1990s?

One of the major problems in studying the rheumatic diseases has been their lack of distinct clinical boundaries and hence the dilemma has been whether or not to lump together apparently heterogeneous entities under a single disease label. The identification, clinically and radiologically, of those subjects whose inflammatory arthritis affected the axial skeleton paved the way for the identification of HLAB27 as the powerful genetic marker for the development of spondyloarthritis. As a consequence a variety of apparently clinically disparate circumstances such as psoriasis and inflammatory bowel disease could usefully be lumped together. By contrast, there has been the recent demonstration of the powerful discriminatory power of specific sequences within the class II region to split different subsets of juvenile arthritis. ${ }^{1}$

The purpose of such attempts to achieve greater homogeneity, either by lumping or splitting, is to improve clinical discrimination, with the hope of better targeting of treatment and more accurate prognostication. Thus one of the major debates in rheumatological therapeutics is on the advantages and disadvantages of early aggressive treatment for rheumatoid arthritis, with much discussion of toppling pyramids. ${ }^{2}{ }^{3}$ The difficulty has been to predict those subjects whose disease would resolve spontaneously and avoid their exposure to unnecessary potentially toxic agents. Clinical and serological status at baseline has been only moderately successful in predicting outcome, and it is claimed that better delineation of the genes, for example in the class II region, might offer a more rational approach to treatment.

\section{MICROCOMPUTERS FOR ALL}

Computers were previously too expensive and too difficult to operate for most individuals. Advances in silicon 'chippery' and the development of even physician friendly software have led to an explosion of personal microcomputers in the past decade, so that it seems possession of such an instrument is as vital to the clinician as his stethoscope. There are considerable advantages but also some hidden dangers. Retrieval of information for clinical research and audit from medical records was a time consuming and often ill rewarded activity because many records were unavailable and, owing to lack of structure, there were problems in obtaining useful information from the remainder. The desire to computerise the important details of presentation and follow up has spawned a myriad of personal clinical databases that will potentially answer efficiently a number of questions about clinical course and response to treatment. The problems are that the uncritical collection of data is seen as an end in itself and lack of standardisation prevents the pooling of results. It is likely, however, that in the next decade there will be an avalanche of publications from individuals and 
groups using this new power to produce larger data sets on outcome and response to intervention than previously available.

Such an exercise requires that any database used is linked to an appropriate statistical package. Statistics have also been made available to the common man with the advent of user friendly statistical software, reducing the apparent need to take expert advice. In some instances this is undoubtedly beneficial. Thus there should now be an acceptance that ' $p$ ' values have little or no value in describing the results of any experiment or study. The emphasis should be on confidence intervals, and the production of easy to use packages for this should enhance the usefulness of data obtained. More powerful statistical tools are also increasingly available and accessible at the touch of a button and will allow all to produce multivariate models for their data without understanding their meaning. There will inevitably be some statistical 'pile-ups' as those used to driving a Metro car find themselves with the power of a Porsche!

$$
\begin{array}{r}
\text { ALAN J SILMAN } \\
\text { The Medical School } \\
\text { University of Manchester } \\
\text { Manchester M13 9PT }
\end{array}
$$

1 Fernandez-Vina $M$ A, Fink C W, Stastny P. DQA 1 and DQB1 alleles in patients with juvenile arthritis (JA) Human Immunology Abstract 1990: 6.2.

2 Wilske $K$ R, Healey L A. Remodeling the pyramid-a concept whose time has come. $\mathcal{J}$ Rheumatol 1989; 16: 565-7.

3 Hess E V, Luggen M E. Remodeling the pyramid-a concept whose time has not yet come. $f$ Rheumatol 1989; 16: 1175-6.

4 Gardner M J, Gardner S B, Winter P D. Confidence interval anlaysis. Microcomputers Program Manual Version 1.0 anlaysis. Microcomputers Program Manual 